\title{
Epidemiologia da esquistossomose em dois municípios da Região Metropolitana de Belo Horizonte, Minas Gerais
}

\section{Epidemiology of schistosomiasis in two municipalities in the metropolitan area of Belo Horizonte, Minas Gerais}

\author{
Raquel Aparecida Ferreira ${ }^{1}$, Stephanie Rezende Faria ${ }^{2}$, \\ Izabella Cristina Alves de Souza ${ }^{3}$, Cristiano Martins da Cruz ${ }^{4}$
}

\begin{abstract}
Resumo
A esquistossomose é uma doença parasitária endêmica em áreas tropicais, apresentando grande importância para a saúde pública no Brasil. Minas Gerais é um dos estados brasileiros que apresenta maior prevalência da doença. Objetivamos descrever os casos de esquistossomose nos municípios de Juatuba e Florestal, Região Metropolitana de Belo Horizonte, Minas Gerais, e identificar o perfil dos infectados. Realizamos consulta às informações contidas nas fichas de notificação da doença, referentes ao período de 2009-2012, fornecidas pelo Setor de Epidemiologia dos dois municípios. Constatamos que no ano de 2011 houve um pico de casos da doença em Juatuba. Observamos um maior número de casos da esquistossomose em indivíduos do sexo masculino e na faixa etária de 1549 anos, em ambos os municípios. No município de Juatuba, os casos de esquistossomose notificados foram, em sua maioria, de moradores residentes em área urbana. Finalmente, constatamos que os cursos hídricos, prováveis focos de esquistossomose, encontram-se em pontos periféricos e distintos, em ambos os municípios. Para o sucesso no controle da doença nos municípios, e em extensão para outros municípios brasileiros, sugerimos a implantação de medidas e ações de prevenção e controle ambiental e educação em saúde para a população.
\end{abstract}

Palavras-chave: Epidemiologia. Esquistossomose. Notificação.

\footnotetext{
${ }^{1}$ Doutorado em Ciências da Saúde (Doenças Infecciosas e Parasitárias) pelo Instituto René Rachou/Fundação Oswaldo Cruz (Fiocruz Minas), Belo Horizonte, Minas Gerais, Brasil. Tecnologista em Saúde Pública do Instituto René Rachou/Fundação Oswaldo Cruz, Belo Horizonte, Minas Gerais, Brasil. E-mail: raapfe@gmail.com

${ }^{2}$ Mestranda no Programa de Pós-graduação em Zoologia da Universidade Federal de Minas Gerais (UFMG), Belo Horizonte, Minas Gerais, Brasil.

${ }^{3}$ Graduação em Ciências Biológicas pelo Centro Universitário UNA, Belo Horizonte, Minas Gerais, Brasil.

${ }^{4}$ Especialista em Saúde Pública pela Escola de Saúde Pública de Minas Gerais (ESP-MG), Belo Horizonte, Minas Gerais, Brasil.
} 


\begin{abstract}
Schistosomiasis is an endemic parasitic disease in tropical areas, of great importance for public health in Brazil. Minas Gerais is one of the Brazilian states with the highest prevalence of the disease. We objectived to describe the cases of schistosomiasis in the municipalities of Juatuba and Florestal, metropolitan region of Belo Horizonte, Minas Gerais, and to identify the profile of those infected. For this, we consulted the information contained in the disease notification forms provided by the epidemiology sector of the two municipalities between 2009-2012. We found that in 2011 the disease peaked in Juatuba. We observed a higher number of cases of schistosomiasis in males aged 15-49 years in both municipalities. In the municipality of Juatuba, most cases of schistosomiasis were reported to be residents of urban areas. Finally, we found that the water courses, probable foci of schistosomiasis, are in peripheral and distinct points in both municipalities. For the successful control of the disease in the municipalities, and in extension to other Brazilian municipalities, we suggest the implementation of measures and actions for prevention and environmental control and health education for the population.
\end{abstract}

Keywords: Epidemiology. Schistosomiasis. Notification.

\section{Introdução}

A esquistossomose é uma doença parasitária que tem como agente etiológico o trematódeo digenético Schistosoma mansoni. ${ }^{(1)}$ Este parasito, em sua fase adulta, vive nas veias do mesentério e do fígado do homem, e na fase juvenil, apresenta como hospedeiro intermediário moluscos do gênero Biomphalaria. ${ }^{(1)}$

A esquistossomose é considerada um importante problema de saúde pública no Brasil, sendo que cerca de 1,5 milhão de pessoas vivem em áreas sob o risco de contrair a infecção. ${ }^{(2)}$ No Brasil, a doença foi descrita em 18 estados e no Distrito Federal, sendo que a ocorrência da doença está diretamente ligada à presença dos moluscos transmissores. ${ }^{(3)}$ Os estados das regiões Nordeste e Sudeste são os mais afetados, agrupando cerca de $99 \%$ dos casos. ${ }^{(3)}$

O processo de descentralização da saúde com a transferência das responsabilidades dos programas de controle de endemias da esfera federal para os estados e municípios ${ }^{(4)}$ foi um marco nos programas de controle de endemias. Neste repasse das responsabilidades (operacionalização, planejamento e execução das atividades de saúde) para os municípios, muitos problemas ocorreram, acarretando, no caso do Programa de Controle da
Esquistossomose (PCE) a sua desarticulação em inúmeras ocasiões, fato que pode ter favorecido o aumento do número de casos da doença em muitos estados brasileiros.

Em Minas Gerais entre os anos de 20072017, a média de notificações da esquistossomose foi de 11.360 casos novos por ano. ${ }^{(5)}$ Atualmente no Estado, há aproximadamente 10 milhões de pessoas vivendo em áreas endêmicas, sendo que em 523 (61\%) dos 853 municípios mineiros há transmissão ativa da esquistossomose. ${ }^{(6)}$ Neste cenário, encontram-se os municípios de Juatuba e Florestal localizados na Região Metropolitana de Belo Horizonte (RMBH), região endêmica para a esquistossomose. ${ }^{(7)}$ Os dois municípios apresentam características semelhantes no que diz respeito a aspectos socioambientais e de situação de saúde, destacando aqui a transmissão da esquistossomose. Estes municípios, que são vizinhos, apresentam fatores biológicos, como presença do hospedeiro intermediário do parasito, presença de ampla variedade de recursos hídricos, todos, fatores de risco para a transmissão da doença e formação de quadros endêmicos. Desta forma, neste estudo objetivamos descrever os casos da doença nos municípios, levantando informações sobre o perfil dos portadores e aspectos epidemiológicos da doença. 


\section{Métodos}

Nós analisamos as fichas de notificação da esquistossomose fornecidas pelos setores de Epidemiologia das Secretarias Municipais de Saúde das cidades de Florestal e Juatuba. Nos municípios, na época avaliada, a notificação dos casos da doença era realizada por médicos, enfermeiros e técnicos das Unidades Básicas de Saúde mais próximas ao local de residência dos usuários. Em seguida, estas fichas eram enviadas ao Setor de Epidemiologia para investigação dos casos e realização do fechamento da notificação.

Apenas no município de Florestal era realizado o inquérito coproscópico anual. Neste caso, os profissionais da Vigilância em Saúde do município entregavam à população potes para coleta das fezes dos usuários, sendo recolhida uma amostra por indivíduo. Este material era repassado aos laboratoristas dos municípios que realizavam os exames das fezes, e constatavam se as amostras eram positivas. Para os indivíduos positivos, o médico realizava o preenchimento da ficha de notificação e o receituário do medicamento. No período avaliado, em Juatuba não era realizado inquérito coproscópico, e assim, provavelmente uma parte dos casos da infecção não foram notificados na população neste período.

Ressalta-se que nas fichas de notificação, que ficam sob a guarda dos Setores de Epidemiologia dos municípios, há mais número de casos registrados e confirmados do que aqueles registrados no Sistema de Informação de Agravos de Notificação (Sinan). Logo, nós optamos por consultar as informações contidas nestas fichas e não no Sinan.

As informações contidas nas fichas de notificação do período de 2009-2012 foram digitadas e sistematizadas em tabelas do software Excel, sendo extraídas as seguintes informações relacionadas ao perfil dos portadores da esquistossomose: faixa etária, sexo, grau de escolaridade e ocupação. Também foi extraída a informação sobre a procedência das contaminações e tipo de recurso hídrico associado, os métodos de diagnóstico utilizados, tipo de tratamento e acompanhamento dos portadores. Ressalta-se que os pesquisadores não tiveram acesso aos nomes, endereços, nem qualquer outro tipo de informação que pudesse identificar os portadores. Desta forma, foram trabalhados dados secundários, semelhante ao disponibilizado publicamente pelo Sinan, sem nenhum tipo de identificação pessoal, não necessitando, o estudo, de submissão ao comitê de ética em pesquisa. Ainda, o estudo teve a anuência dos representantes das secretarias de saúde municipais para o seu desenvolvimento.

Além da análise das informações contidas nas planilhas do Excel fornecidas pelos municípios, foi solicitado aos coordenadores locais dos serviços informações adicionais, tais como: possíveis locais de contaminação dos portadores, se a residência dos mesmos era em área urbana ou rural, se haviam tomado a medicação corretamente, quais foram as orientações passadas sobre a doença aos portadores pelos profissionais de saúde, e quais eram as formas de controle empregadas pelas autoridades sanitárias nos municípios.

As informações relacionadas ao número de casos confirmados da doença, faixa etária dos portadores, sexo e grau de escolaridade dos portadores em função dos anos foram processadas e confeccionados gráficos por meio do programa SigmaPlot, versão 8.0

Todos os locais de contaminação discriminados pelos profissionais tiveram as coordenadas geográficas coletadas através de GPS marca Garmin eTrex; posteriormente foram confeccionados mapas utilizando i3Geo - software livre, licenciado como GPL (GNU General Public License - Licença Pública Geral), criado pelo Ministério do Meio Ambiente (MMA), versão 4.6. Não foi possível confirmar se havia focos de transmissão da doença nas coleções hídricas mencionadas nas notificações pelos portadores e pelos coordenadores, uma vez que nos municípios não era realizada pesquisa malacológica nas coleções hídricas à época do estudo. 


\section{Resultados}

Entre o período de 2009 a 2012 foram confirmados 43 casos de esquistossomose em Florestal e 35 casos em Juatuba (Figura 1). Em Florestal, o número de casos confirmados da doença mostrou-se pouco variável entre os anos de 2009 a 2011. Entretanto, no ano de 2012 observou-se uma queda no número de casos no município: apenas dois casos confirmados (Figura 1).

Em Juatuba, não houve aumento no número de casos da doença em 2009, 2010 e 2012, mantendose cinco casos anuais (Figura 1). Entretanto, em 2011, houve um aumento expressivo no número de notificações, quando foram registrados 20 casos confirmados da doença (Figura 1).

Figura 1 - Casos confirmados de esquistossomose notificados em Florestal e Juatuba no período de 2009-2012.

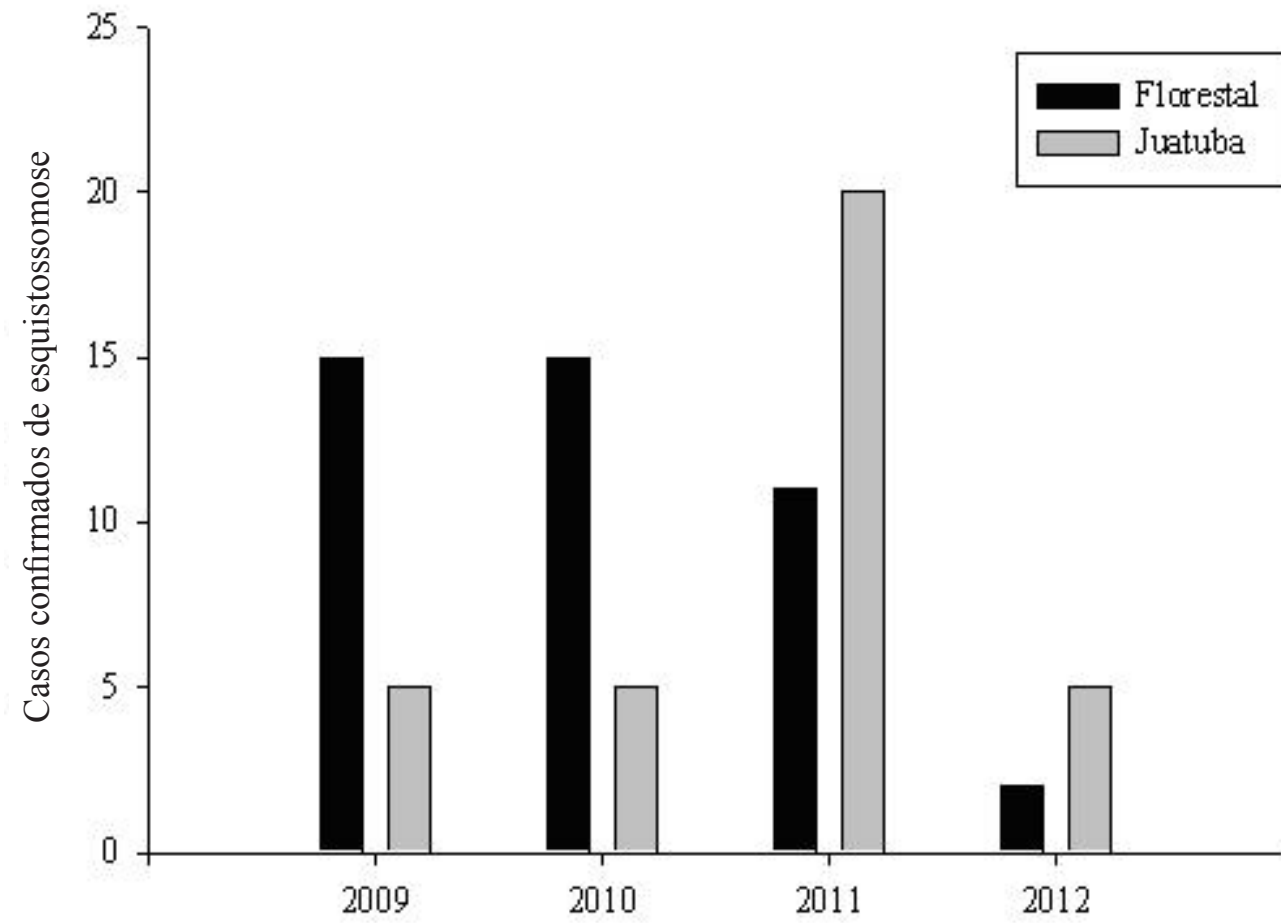

Fonte: Autores

A distribuição dos casos de esquistossomose em função da faixa etária foi bastante semelhante em ambos os municípios (Figura 2). Observou-se que na faixa etária dos 15-29 anos houve 27,9\% de casos confirmados da doença em Florestal e $20 \%$ em Juatuba. Na faixa dos 30-49 anos esta porcentagem foi de 46,5\% em Florestal e 48,6\% em Juatuba (Figura 2). Também foi observado um menor no número de casos nas faixas etárias posteriores em ambos os municípios (Figura 2).

Em relação ao sexo, evidenciou-se que nos dois municípios o maior número de casos confirmados ocorreu no sexo masculino $(65,1 \%$ em Florestal e 77,1\% em Juatuba) (Figura 3).
Em relação à escolaridade dos portadores, muitas notificações não continham registros sobre esta informação $(53,5 \%$ das fichas de Florestal e $28,5 \%$ das fichas de Juatuba). Ainda, grande parcela das fichas estava preenchida como escolaridade ignorada (41,9\% em Florestal e $25,7 \%$ em Juatuba), restando apenas 2,9\% de fichas preenchidas com ensino fundamental incompleto e $2,9 \%$ ensino fundamental completo em Florestal (Figura 4) e 20\% ensino fundamental incompleto, $11,4 \%$ ensino fundamental completo, $2,9 \%$ ensino médio incompleto, $8,6 \%$ ensino médio completo e $2,9 \%$ ensino superior completo em Juatuba (Figura 4). 
Figura 2 - Porcentagem de casos confirmados de esquistossomose em função da faixa etária em Florestal e Juatuba no período de 2009-2012.

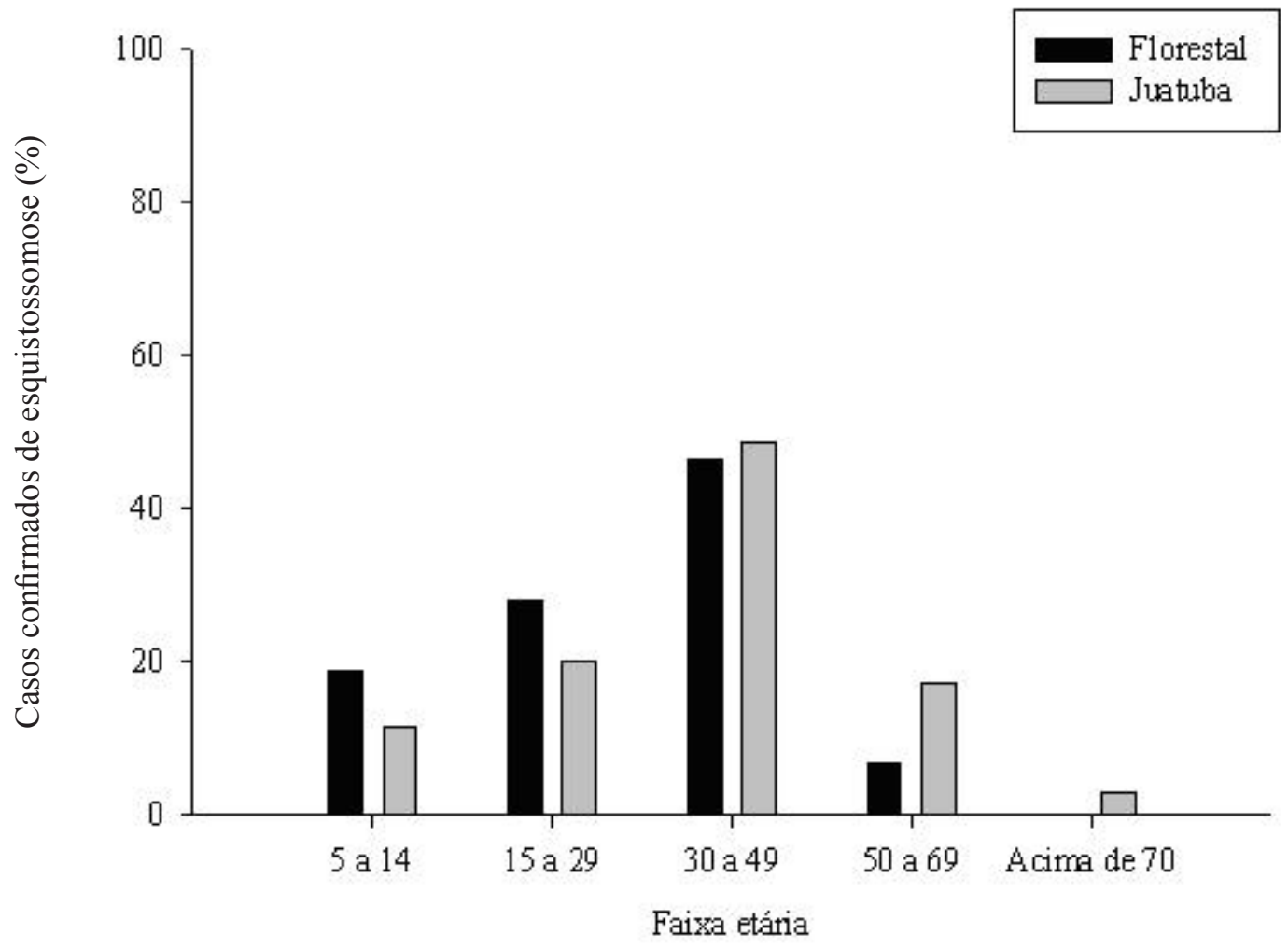

Fonte: Autores

Figura 3 - Porcentagem de casos confirmados de esquistossomose em função do sexo em Florestal e Juatuba no período de 2009-2012.

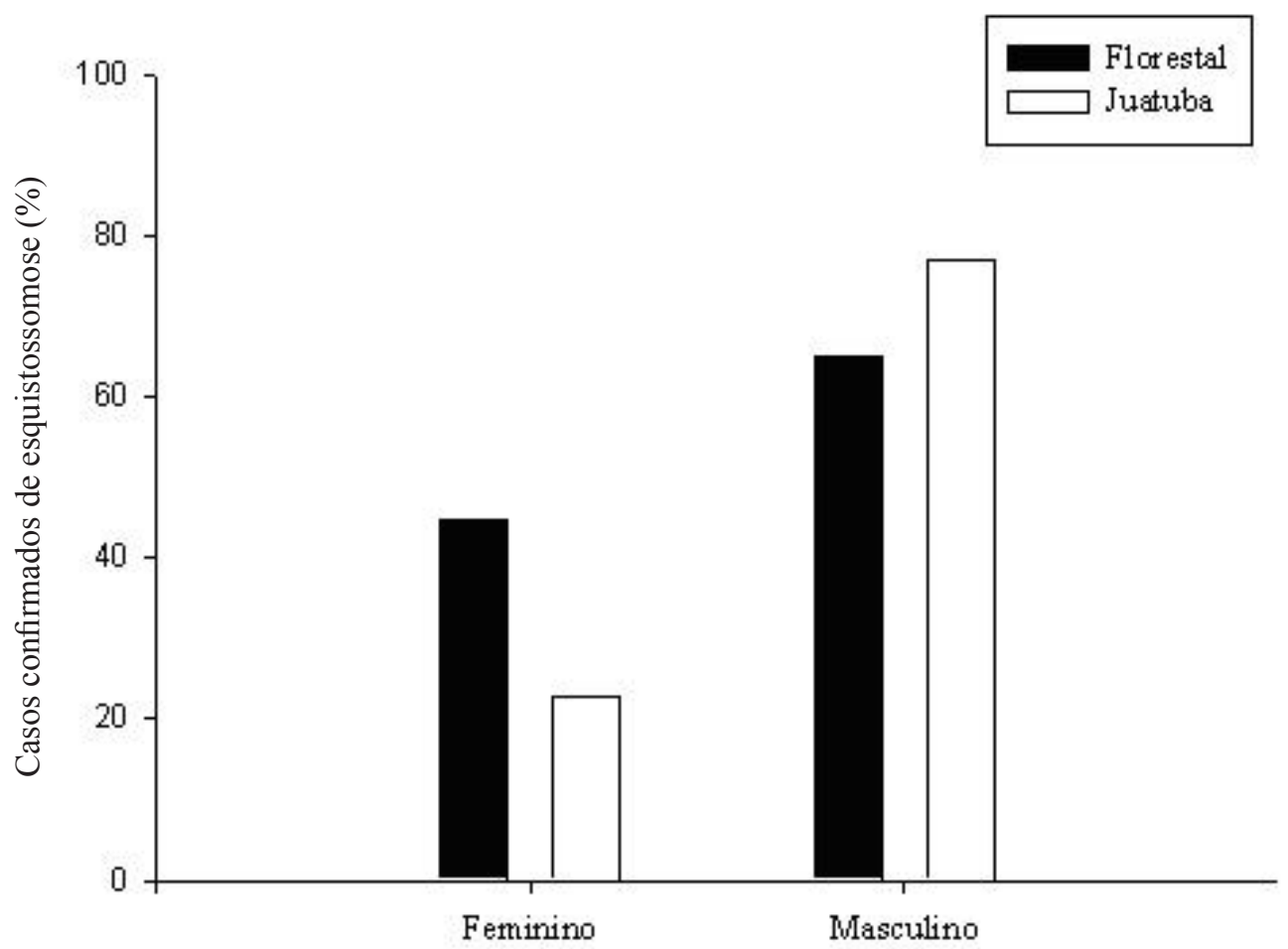

Fonte: Autores 
Figura 4 - Porcentagem de casos confirmados de esquistossomose em função da escolaridade em Florestal e Juatuba no período de 2009-2012.

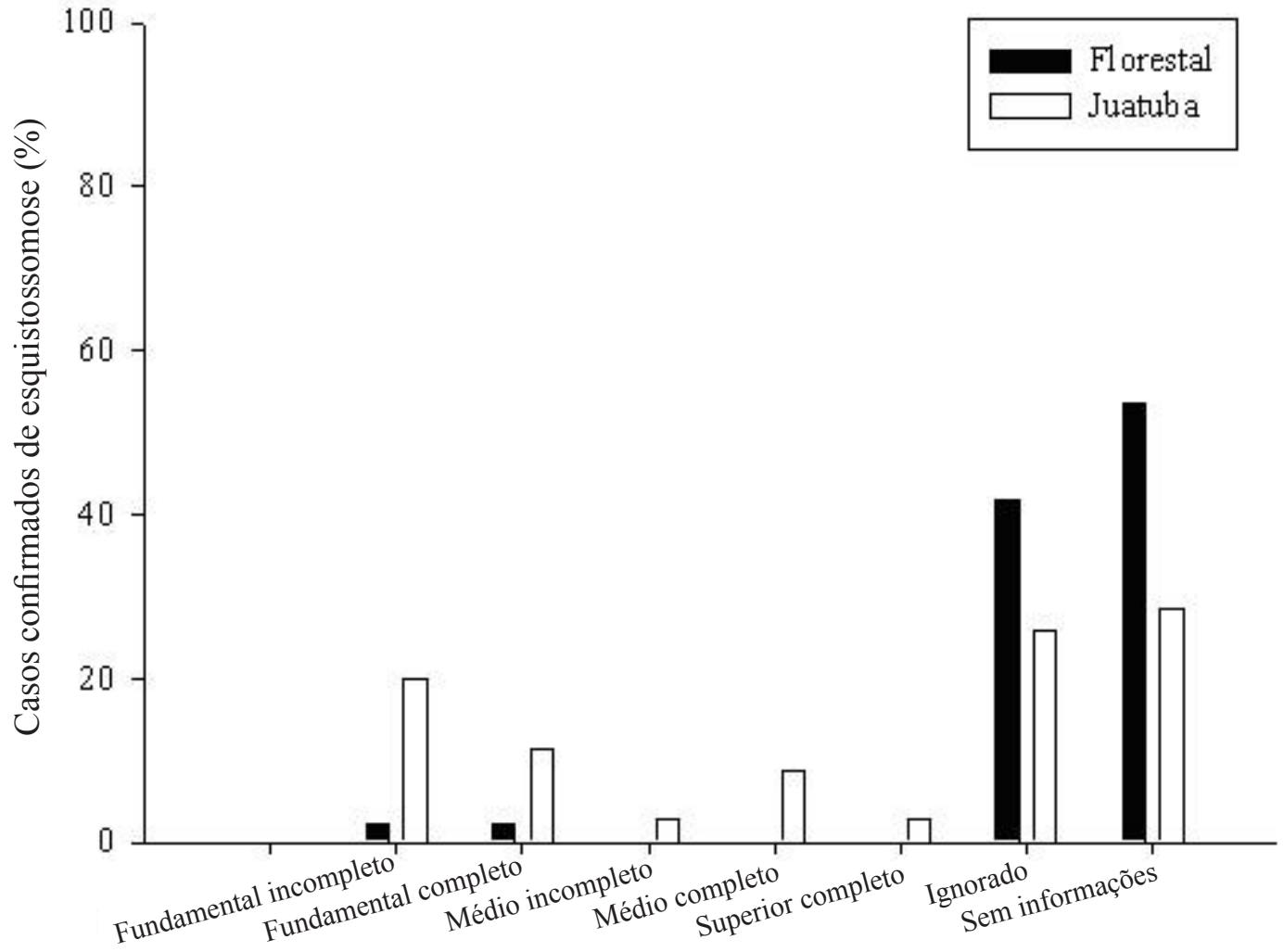

Fonte: Autores

As fichas de notificação dos municípios continham informações importantes sobre $\mathrm{o}$ diagnóstico e tratamento dos portadores. O exame parasitológico usado pelos laboratórios contratados pelas secretarias municipais de saúde dos municípios era o Método de Hoffman, Pons e Janer (HPJ).

Todos os portadores tiveram como forma clínica da doença assinalada a intestinal, e foram tratados com o medicamento Praziquantel $600 \mathrm{mg}$. Segundo a coordenadora da Vigilância em Saúde de Juatuba, no ato do diagnóstico o portador era orientado quanto à doença e a forma de administração da medicação, que é dose única. Os portadores também eram orientados quanto às possíveis reações adversas, sendo aconselhados a ficarem em repouso por 3 horas após ingestão do medicamento para minimizar possíveis desconfortos advindos da medicação. O serviço de Vigilância em Saúde da cidade de Florestal realizava também as mesmas ações junto aos portadores.
Quanto à evolução clínica dos casos, em Juatuba houve 31 notificações registradas como "cura" e quatro notificações registradas como "sem informação". Diferentemente de Florestal que teve 12 notificações registradas como "cura" e 31 como "sem informação".

No município de Juatuba os casos de esquistossomose foram, em sua maioria, de moradores residentes em área urbana (Figura 5). Algumas notificações em Juatuba assinalaram que os portadores se contaminaram, provavelmente, em diversas coleções hídricas em bairros do próprio município, como, por exemplo, no ribeirão Serra Azul e em lagoas da Fazenda Eldorado, ambos no distrito de Francelinos (Figura 5). Além de coleções hídricas de outros municípios, como cachoeiras da região do município de Itaúna, lagoas na cidade de Ribeirão das Neves, ribeirões e lagoas na comunidade rural de Ribeirão do Ouro em Florestal e no Rio São Francisco nas imediações da cidade de Três Marias. 
Figura 5 - Mapa georreferenciado das coleções hídricas, prováveis focos de transmissão da esquistossomose nos municípios de Florestal e Juatuba.
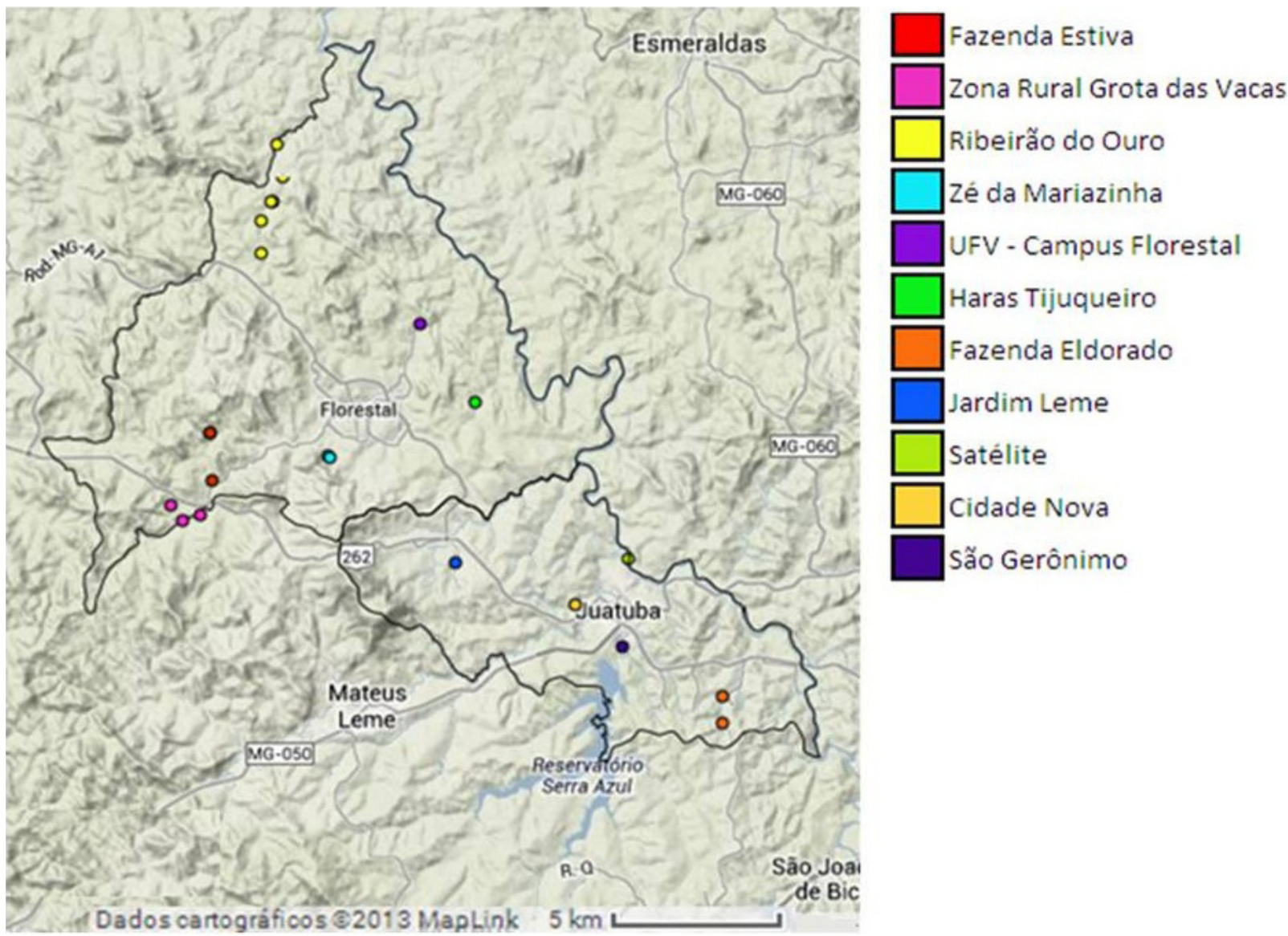

Fonte: Autores

Em Florestal, a maioria das notificações era de moradores da área rural, apesar de haver algumas notificações de residentes da área urbana. Os locais de contaminação declarados pelos portadores de Florestal, e que possivelmente foram os focos da infecção, foram as seguintes coleções hídricas do município: uma lagoa na fazenda Estiva, um ribeirão na zona rural da localidade de Grota das Vacas, ribeirões e lagoa em Ribeirão do Ouro, também área rural de Florestal, uma lagoa na fazenda do Zé da Mariazinha, lagoas do Campus da Universidade Federal de Viçosa em Florestal e uma lagoa no Haras Tijuqueiro, também região rural de Florestal (Figura 5).

No período analisado, também houve 30 notificações nas quais não era mencionada nenhuma informação sobre os prováveis focos de contaminação.

\section{Discussão}

Entre os anos de 2009-2012 o programa de controle da esquistossomose não esteve implantado efetivamente nos municípios de Florestal e Juatuba, de forma que não foram realizadas pesquisas malacológicas periódicas para captura, exame de positividade, identificação, registro e classificação taxonômica dos planorbídeos presentes na região. Tampouco foram realizadas ações educativas voltadas para a população das áreas suspeitas de transmissão, registro de informações da "dinâmica" da doença e inquérito coproscópico para detecção e tratamento de novos portadores da doença no município de Juatuba, ações estas realizadas no município de Florestal. A ausência total de ações do programa de controle da esquistossomose em Juatuba provavelmente é uma das causas do 
aumento expressivo do número de casos da doença no ano de 2011. Por outro lado, o decréscimo no número de casos da doença no ano de 2012 em Florestal provavelmente se deve às ações de controle empregadas, mesmo que de forma incipiente, e também às orientações constantes sobre a doença e sua profilaxia feitas por meio de ações educativas junto aos moradores das áreas onde ocorreu o maior número de casos confirmados.

A análise da incidência da doença não foi realizada neste estudo. Apesar disto, chama a atenção o registro de maior número de casos da esquistossomose nas faixas etárias de 30-39 anos e de 15-29 anos, fato que está de acordo com outros estudos realizados. Um trabalho realizado no município de Jaboatão dos Guararapes, Pernambuco, verificou que a positividade em adultos era superior na faixa etária entre 20-59 anos, ${ }^{(8)}$ o mesmo sendo observado no estado do Paraná. (9) Possivelmente, o acometimento de faixa etária economicamente produtiva está relacionado com alguns hábitos da população, como o contato com a água nas ocasiões de lazer, ou mesmo em atividades de trabalho. ${ }^{(10-11)}$

Em Florestal foi registrado um maior número de casos da doença na faixa de 5-14 anos comparado a Juatuba, provavelmente porque em Florestal era realizado inquérito coproscópico junto à população, evidenciando assim a importância desta ação na detecção de novos casos da doença.

Conforme esperado, o maior número de casos da doença ocorreu no sexo masculino, conforme já demonstrado em estudos anteriores. ${ }^{(8,12-13)}$ Uma pesquisa realizada no município de Santo Antônio de Jesus, Bahia, entre os anos de 2014 e 2016, verificou que 58,6\% dos indivíduos acometidos pela doença eram do sexo masculino. ${ }^{(12)}$ Em outro estudo realizado no município de Sabará, Região Metropolitana de Belo Horizonte, entre os anos de 2012-2014 também foi demonstrado um maior acometimento da doença em crianças e adultos jovens, ambos do sexo masculino. ${ }^{(13)}$ Conforme já discutido acima, a maior prevalência da doença em indivíduos jovens e do sexo masculino possivelmente está relacionada ao hábito destes indivíduos, que usam os cursos hídricos para atividades de lazer, banhos, pescas e práticas esportivas, além de atividades profissionais como lavoura ou pesca. ${ }^{(13)}$

Devido à ausência de dados nas fichas de notificação da doença relacionados à escolaridade dos portadores, em ambos os municípios, não foi possível descrever se há uma escolaridade específica dos portadores associada a um maior número de casos da doença. Um estudo realizado nas localidades de Forte Orange e Serrambi no estado de Pernambuco apontou que a maior porcentagem dos casos de contaminação provinha de indivíduos que apresentavam ensino fundamental incompleto

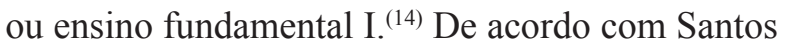
et al., ${ }^{(15)}$ a baixa escolaridade é um fator de risco tanto para a transmissão como contaminação da esquistossomose. Em outras palavras, um indivíduo com menor grau de escolaridade vai estar mais suscetível a contrair a doença por não ter tido uma educação de base concisa. ${ }^{(15)}$

Por meio do presente estudo foi possível constatar que o exame parasitológico de fezes usado pelos laboratórios contratados pelas secretarias municipais de saúde ainda é um dos métodos mais utilizados na rotina laboratorial ou inquéritos coproparasitológicos. ${ }^{(16-17)}$ Ressaltase que o método HPJ é barato, o que para os municípios é muito importante, já o método de Kato-Katz tem um custo mais elevado. Por outro lado, o Kato-Katz é um método mais sensível para pesquisas de ovos de $S$. mansoni, revelando um maior número de casos positivos. ${ }^{(1)}$ Desta forma, alguns casos podem não ter sido confirmados nos municípios, especialmente em Florestal, que era realizado inquérito coproscópico. Este fato é agravado pelo fato de que apenas uma amostra era colhida por paciente.

Em relação ao tratamento dos portadores com o Praziquantel, Marculino et al. ${ }^{(18)}$ afirmam que esse medicamento atua sobre todas as espécies do gênero Schistosoma, lesando o tegumento do parasito, e facilitando a exposição deste a resposta imune do hospedeiro. Em um estudo realizado por Emanuel e Prata, ${ }^{(19)}$ avaliando a eficácia 
do Praziquantel, $83 \%$ dos portadores tratados foram curados após o tratamento. O Praziquantel é o único fármaco disponível, distribuído gratuitamente pelo Ministério da Saúde aos estados e municípios..$^{(1)}$ Ainda sobre o tratamento, destaca-se que os portadores receberam as devidas orientações relacionadas à ingestão e efeitos da medicação.

É de fundamental importância a realização do acompanhamento do critério de cura do portador. Esse fluxo de serviço foi realizado no município de Juatuba no período avaliado, estando de acordo com o preconizado pelo Ministério da Saúde. ${ }^{(20)}$ Entretanto, esta ação não foi realizada no município de Florestal, demonstrando falha no processo de controle e prevenção da doença, uma vez que o portador tratado pode ter permanecido positivo e, consequentemente, fonte de infecção e disseminação do parasito.

Os cursos hídricos, prováveis focos de esquistossomose, encontravam-se em pontos periféricos e em diferentes regiões, em ambos os municípios, o que provavelmente potencializou a contaminação da população, aumentando as chances de um maior número de pessoas entrarem em contato com as águas. Estudos futuros poderão avaliar se houve ocupação desordenada próxima a esses cursos, e se isto criou as condições ambientais ideais para o surgimento de criadouros dos moluscos vetores em locais propícios para a infecção humana, estabelecendo a transmissão da doença. Segundo Silva ${ }^{(21)}$ as alterações em ecossistemas primitivos podem ser consideradas como o fator epidemiológico condicionante que propicia a incorporação da doença nas paisagens. Além disto, os cursos hídricos, prováveis focos de esquistossomose, devem ser priorizados em futuras pesquisas malacológicas, e quando encontrados os hospedeiros intermediários infectados, estes cursos devem ser interditados paro uso pela população. Os resultados do inquérito malacológico poderiam, também, esclarecer se a transmissão da esquistossomose nas áreas é específica de um período climático ou se ocorrem ao longo de todo o ano.
Em Juatuba, no período avaliado, a maioria dos portadores da esquistossomose residia em área urbana, ao contrário de Florestal. Ainda, em Juatuba, grande parte dos prováveis locais de contaminação foram coleções hídricas localizadas em área urbana. Uma pesquisa realizada em Pernambuco identificou áreas de risco de transmissão urbana da esquistossomose, estabelecendo maior risco de transmissão para a população. ${ }^{(22)}$ Além dos casos notificados em regiões urbanas do Nordeste do País, pesquisas na Região Norte também mostraram uma maior porcentagem de casos de contaminação da doença em áreas urbanas, 71\%.. ${ }^{(23)}$ Tal pesquisa afirma que os indivíduos contaminados provavelmente exerciam atividades rurais e/ou atividades de ecoturismo, fato de grande relevância na epidemiologia da doença. ${ }^{(23)}$ No período avaliado, a população de Juatuba esteve bastante exposta ao risco de contrair a infecção, uma vez que, conforme relatado pelos portadores, provavelmente existiam no município coleções hídricas urbanas contaminadas pelo parasito causador da doença. Em Florestal, as contaminações provavelmente ocorreram em área rural, conforme relatado pelos portadores, o que provavelmente está relacionadoàs condições precárias socioambientais. ${ }^{(24-25)}$ Apesar de não haver relatos de contaminações em áreas urbanas de Florestal, não se descarta a existência de focos urbanos da doença. Esta confirmação seria possível se as pesquisas malacológicas estivessem sendo realizadas nos municípios no período avaliado neste estudo. No entanto, com a desarticulação do Programa de Controle da Esquistossomose (PCE) muitos municípios não possuem os insumos e equipamentos laboratoriais necessários para as atividades de pesquisa malacológica. Soma-se a escassez de agentes de endemias, trabalhando no PCE.

\section{Conclusão}

A esquistossomose é um problema de saúde nos municípios de Florestal e Juatuba. Ainda, ambos os municípios apresentavam condições 
ambientais e sociais propícias à manutenção e ampliação dos focos da doença à época do estudo.

A esquistossomose teve um pico de casos confirmados em Juatuba no ano de 2011, ocorrendo um maior número de casos notificados em indivíduos na faixa etária de 15-29 anos e no sexo masculino em ambos os municípios. Além disso, foi possível descrever as formas de diagnóstico, tratamento e acompanhamento dos portadores adotados pelo sistema de saúde dos municípios. No entanto, informações relacionadas à escolaridade e ocupação dos portadores não foram analisadas, uma vez que as informações presentes nas fichas de notificação foram deixadas em branco ou ignoradas. O preenchimento inadequado das fichas de notificação evidencia a possível ausência de ações de sensibilização e capacitação dos profissionais para a importância deste serviço, fazendo, provavelmente, com que eles o entendam, apenas como uma atividade protocolar. $\mathrm{O}$ melhor preenchimento desses formulários provavelmente ocorreria se no município fossem realizadas sensibilizações, capacitações e cursos direcionados ao tema.

Percebemos que um dos fatores mais importantes relacionados ao problema da expansão da doença na região era ausência de um programa de controle da doença bem estruturado e atuante. Devido a esta lacuna, na época do estudo, não haviam pesquisas malacológicas periódicas para captura, exame de positividade, identificação, registro e classificação taxonômica dos vetores do agente presente na região. No município de Juatuba não estavam implementadas ações educativas destinadas à população exposta à infecção, e nem inquérito coproscópico para detecção e tratamento de novos portadores. Por sua vez, em Florestal havia falha no acampamento do paciente tratado.

A esquistossomose é uma doença que apresenta alta complexidade epidemiológica, o ciclo e o mecanismo de transmissão do parasito, aliada à diversidade de fatores condicionantes dificultam o controle da doença pelos serviços de saúde. ${ }^{(26)}$ Em áreas endêmicas, a instalação de formas graves da doença podem acontecer em virtude do caráter assintomático da doença que muitas vezes apresenta evolução silenciosa no indivíduo, sendo uma doença que exige monitoramento constante. ${ }^{(27)}$ Ainda, casos de esquistossomose aguda em área endêmica são raros, mas ainda assim, provavelmente são negligenciados, mal diagnosticados, subestimados e subnotificados. (26) Estes fatores, associados à escassez de recursos financeiros, falta de pessoal, ausência de capacitações e educação permanente dos profissionais de saúde, deficiência no controle ambiental e esparsas ações de educação em saúde para população tornam o controle da doença um desafio. Este é o caso nos municípios estudados, e provavelmente a realidade para outros municípios de Minas Gerais e em extensão do Brasil, pois mesmo existindo esforços dos serviços de saúde nos municípios, há dificuldades na execução de ações básicas direcionadas ao controle da doença. Desta forma, sugerimos que a melhoria das condições socioeconômicas, culturais e ambientais, grandes determinantes sociais de saúde, somados à melhoria no planejamento e definição de prioridades nas ações em saúde municipais, geraria melhoria nas condições de prevenção e controle da esquistossomose nos municípios estudados.

\section{Agradecimentos}

Agradecemos às Secretarias Municipais de Saúde de Florestal e Juatuba pelo fornecimento dos dados, e também pela disponibilidade e atenção dos profissionais de saúde dos municípios.

\section{Referências}

1 Ministério da Saúde. Secretaria de Vigilância em Saúde. Guia de Vigilância em Saúde. Volume 3. 1a ed. atual. Brasília: Editora do Ministério da Saúde; 2017.

2 Brasil. Ministério da Saúde. Saúde de A a Z. Esquistossomose: causas, sintomas, tratamento, diagnóstico e prevenção [Internet]. Brasília: Ministério da Saúde. 2020 [citado 2020 Jun. 23]. Disponível em: https://saude.gov.br/saudede-a-z/esquistossomose 
3 Ministério da Saúde. Secretaria de Vigilância em Saúde. Coordenação-Geral de Desenvolvimento da Epidemiologia em Serviços. Guia de Vigilância em Saúde. Brasília: Ministério da Saúde; 2016.

4 Brasil. Ministério da Saúde. Portaria no 1.399, de 15 de dezembro de 1999. Regulamenta a NOB SUS 01/96 no que se refere às competências da União, estados, municípios e Distrito Federal, na área de epidemiologia e controle de doenças, define a sistemática de financiamento e dá outras providências [Internet]. Brasília: Ministério da Saúde; 1999. [citado 2019 ago 7]. Disponível em:// oemmndcbldboiebfnladdacbdfmadadm/http:// www.funasa.gov.br/site/wp-content/files_mf/ Pm_1399_1999.pdf

5 Carvalho AX Filho, Barbosa LGC, Passos RPS, Maia JJS. Doenças negligenciadas em Minas Gerais e determinantes sociais. Atenas Higéia 2019;1(1):1-6.

6 Drummond SC, Pereira SRS, Silva LCS, Antunes C, Figueiredo M, Lambertucci JR. Schistosomiasis control program in the state of Minas Gerais in Brazil Mem. Inst. Oswaldo Cruz. 2010;105(4).

7 Katz N, Peixoto SV. Análise crítica da estimativa do número de portadores de esquistossomose mansoni no Brasil. Rev. Soc. Bras. Med. Trop. 2000;33(3):303-8. doi: 10.1590/S003786822000000300009 .

8 Gomes ACL, Galindo L, Natália N, Silva EVG. Prevalência e carga parasitária da esquistossomose mansônica antes e depois do tratamento coletivo em Jaboatão dos Guararapes, Pernambuco. Epidemiol Serv. Saúde 2016; 25(2):243-50. doi: 10.5123/s167949742016000200003.

9 Costa Al, Daiane SB, Tatiane SG, Reinaldo M, Flávia TRS, Simone CCSM, et al. Esquistossomose Urbana no Norte Pioneiro do Estado do Paraná, Brasil. J. health. sci. 2018;19(4):251-5.
10 Oliveira ALL, Anamaria MMP, Maria ECD, Elisa TO, Cássia RL, Marcos S, et al. Foco emergente de leishmaniose visceral em Mato Grosso do Sul. Rev Soc Bras Med Trop. 2006;39(5):446-50. doi: 10.1590/S003786822006000500005 .

11 Vasconcelos $\mathrm{CH}$, Cardoso PCM, Quirino WC, Massara CL, Amaral GL, Cordeiro R, et al. Avaliação de medidas de controle da esquistossomose mansoni no Município de Sabará, Minas Gerais, Brasil, 1980-2007. Cad Saúde Pública 2009; 25(5):997-1006. doi: 10.1590/S0102-311X2009000500006.

12 Silva, STB. Prevalência da esquistossomose entre os participantes notificados no programa de controle da esquistossomose no município de Santo Antonio de Jesus-BA [trabalho de conclusão de curso]. Governador Mangabeira: Faculdade Maria Milza; 2017.

13 Santos LP, Nogueira MJ, Rezende CN, Ferreira RA. Doenças negligenciadas no município de Sabará: casos, portadores e percepções. Arq. Cienc. Saúde UNIPAR. 2017; 21(3):155-62. doi: 10.25110/arqsaude.v21i3.2017.5860.

14 Barbosa VS. Análise da paisagem de risco para transmissão da esquistossomose em localidades do litoral de Pernambuco [tese de doutorado]. Recife: Fundação Oswaldo Cruz; 2017.

15 Santos GP, Oliveira HMB, Oliveira AA Filho. Educação ambiental em saúde: análise dos casos de esquistossomose notificados na Paraíba no período de 2015 a 2017 [Internet]. Educ amb. ação. 2018 [citado 2019 ago 6]; 64(17). Disponível em: http://www.revistaea. org/artigo.php?idartigo $=3528$

16 Tibiriça SHC, Abramo C, Simões AS, Pinheiro, IO, Ribeiro LC, Coimbra ES. Validação do número de lâminas para realização do método de sedimentação espontânea das fezes. $\mathrm{Hu}$ Revista 2009;35(2).

17 NevesEVR.Prevalência de enteroparasitoses em crianças do ensino fundamental I, matriculadas na escola Manoel Machado Pedreira, no município de Governador Mangabeira-BA [trabalho de conclusão de curso]. Governador Mangabeira: Faculdade Maria Milza; 2018. 
18 Marculino HHS, Nascimento KP, Pacheco RA, Sampaio MGV. Esquistossomose: uma questão de saúde pública. Mostra Interdisciplinar do curso de Enfermagem. 2017; 2(1).

19 Emanuel A, Prata A. Comparação entre praziquantel e oxamniquine no tratamento da esquistossomose mansoni. Rev. Soc. Bras. Med. Trop. 1983;16(2):90-3. doi: 10.1590/ S0037-86821983000200005.

20 Brasil. Ministério da Saúde. Secretaria de Vigilância em Saúde Departamento de Vigilância Epidemiológica. Vigilância da Esquistossomose Mansoni: diretrizes técnicas/ Ministério da Saúde, Secretaria de Vigilância em Saúde, Departamento de Vigilância das Doenças Transmissíveis. - 4. ed. - Brasília: Ministério da Saúde, 2014.

21 Silva LJ. O conceito de espaçona epidemiologia das doenças infecciosas. Cad. Saúde Pública 1997; 13(4):585-93. doi: 10.1590/S0102311X1997000400002.

22 Gomes ECS, Mesquita MCS, Rehn VNC, Nascimento WRC, Loyo R, Barbosa CS. Urban transmission of schistosomiasis: new epidemiological situation in the forest area of Pernambuco. Rev. Bras. Epidemiol. 2016;19(4):822-34. doi: 10.1590/19805497201600040012 .

23 Rodrigues CA Junior, Dias FCF, Rosa RTAS, Cardozo CRL, Veloso FPFS, Mariano SMB, et al. Esquistossomose na região norte do Brasil. Rev. Patol. Toc. 2017; 4(2): 58-61. doi: 10.20873/uft.2446-6492.2017v4n2p58.

24 Barbosa CS, Barbosa FS. Padrão epidemiológico da esquistossomose em comunidade de pequenos produtores rurais de Pernambuco, Brasil. Cad. Saúde Públ. 1998;14(1):129-37. doi: 10.1590/S0102311X1998000100020.

25 Leal OB Neto, Galvão TYC, Esteves FAM, Gomes AMAS, Gomes ECS, Araújo KCGM, et al. Análise espacial dos casos humanos de esquistossomose em uma comunidade horticultora da Zona da Mata de Pernambuco, Brasil. Rev. Bras. Epidemiol. 2012;15(4): 77180. doi: 10.1590/S1415-790X2012000400009.
26 Lambertucci JR. Acute schistosomiasis mansoni: revisited and reconsidered. Mem Inst Oswaldo Cruz 2010; 105(4): 422-35. doi: 10.1590/S0074-02762010000400012.

27 Quites HFO, Abreu MNS, Matoso L, Ferreira, GA. Avaliação das ações de controle da esquistossomose na Estratégia de Saúde da Família em municípios do Vale do Jequitinhonha em Minas Gerais. Rev. Bras. Epidemiol. 2016; 19(2): 375-89. doi: 10.1590/1980-5497201600020014.
Recebido em: 31 maio 2019

Aceito em: 23 ago. 2019 